\title{
Investigation of the modular building metal frame nodal connection rotational stiffness
}

\author{
Vyacheslav Shirokov, Oleg Veremeenko, and Vadim Alpatov* \\ Samara State Technical University, 443100, Samara, Russia
}

\begin{abstract}
The research subject is the rotational stiffness of the modular building metal frame nodal connection. The stiffness of nodal connections has a significant impact on the results of calculating the load-bearing frame of a modular building. It is known that absolutely rigid connections exist only in theory. In practice, each nodal connection has a finite stiffness, as evidenced by numerous studies of the researchers. When designing the real objects, the studies on the stiffness of nodal connections of a certain design should be carried out. As a rule, when designing real objects, there is no time to carry out the research. The designer operates with well-known and reliably proven recommendations. It is important for him to have the ready-made solutions for implementation in design practice. Such solutions can be provided on the basis of preliminary studies of the joints' behavior of the structural elements under load. The article presents a solution to such a problem. The study of the stiffness of the nodal connection with the connected elements parameters variation has been carried out. The purpose of the study is to find the boundaries of design solutions at which the connection can be considered rigid.
\end{abstract}

\section{Introduction}

The classification of nodal connections is adopted according to Eurocode 3, which sets the boundaries for hinged, semi-rigid and rigid nodes. To calculate the initial rotational stiffness values, the component finite element method was used (CFEM), implemented in IDEA StatiCA. As a result of the study, the rotational stiffness values of fastening the crossbars to the columns have been obtained. It has been established that, in the spans typical for modular buildings, the nodes with direct adjoining channels to square tube racks should be generally classified as semi-rigid and their rotational stiffness should be taken into account in the design schemes. For the nodes with an additional rib, the change in the attachment rotational stiffness is non-linear.

Based on the research results, a nomogram for various sections of channels and square pipes with minimum rib sizes, at which the crossbar-to-rack connection node should be considered rigid, has been built. This nomogram is recommended for use when dimensioning stiffeners.

\footnotetext{
*Corresponding author: avu75@mail.ru
} 
Currently, the construction of modular buildings from steel structures is an important trend in the construction industry. According to literary sources, prefabricated modular buildings (PMB) - these are the structures assembled from the volumetric unified elements prefabricated block-modules, including the systems of internal engineering equipment that provide the specified physical and mechanical properties of structures, stability, stiffness, strength, invariability of the geometric dimensions of modules during their transportation and installation" [1-4]. Such buildings are the permanent structures, often with several floors. They are widely used in the development of territories on a rotational basis and in remote regions. By designation, such buildings can be: residential; public; industrial. The latter can be made fully equipped in accordance with their purpose, that is, have built-in boiler equipment, transformer equipment, etc. In such cases, modular buildings can have an increased level of responsibility.

The frame of one modular block usually consists of vertical columns, to which horizontal frames are attached (Fig. 1). To ensure stiffness and geometric invariability, the connection between the crossbars and the columns is extremely important [5].

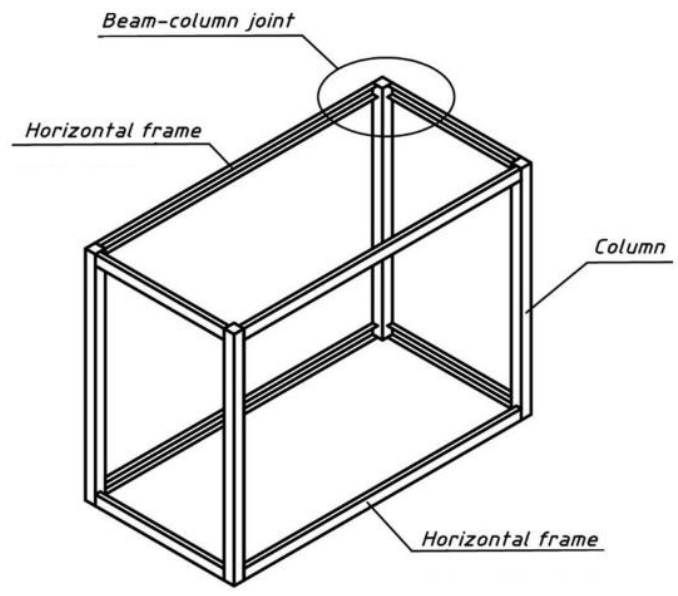

Fig. 1. Scheme of the modular block frame

When calculating modular buildings, the junction of horizontal frames and racks can be assumed to be rigid or semi-rigid. The stiffness of the fastening depends on the joint and the elements' design is to be connected. Taking into account the stiffness of the elements' nodal connection has a significant impact on the forces' distribution in the modular buildings' frame $[5,6]$. At the same time, in design practice, they tend to use exactly rigid nodes. This is due to the difficulties in placing connections within the frame of a modular building. Due to this complexity, the designers strive to give the maximum possible stiffness to the frame elements' joints, to ensure the geometric immutability and overall stiffness of the module "on their own".

It is known that absolutely rigid connections exist only in theory. In practice, each nodal connection has a finite stiffness, as evidenced by numerous researches works [7-9]. When designing real objects, the studies of the stiffness of nodal connections of a certain design should be carried out. As a rule, when designing real objects, there is no time to carry out the research. The designer operates with well-known and reliably proven recommendations. It is important for him to have the ready-made solutions for implementation in design practice. Such solutions can be provided on the basis of preliminary studies of the joints' behavior of structural elements under load. The following is a solution to this problem. The study of the nodal connection stiffness with variation of the connected elements' parameters 
has been carried out. The purpose of the study is to find the boundaries of design solutions at which the connection can be considered rigid.

\section{Materials and methods}

In construction practice, various solutions for nodal connections of modular buildings elements are common [10-13]. One of the most common solutions is the direct welding of girders from the channels to columns made of square or rectangular pipes (Fig. 2.a) [14-18]. Such a node may have an additional stiffening rib (Fig. 2.b). The subject of this research is the study of the rotational stiffness of the specified nodal connection of a modular building.
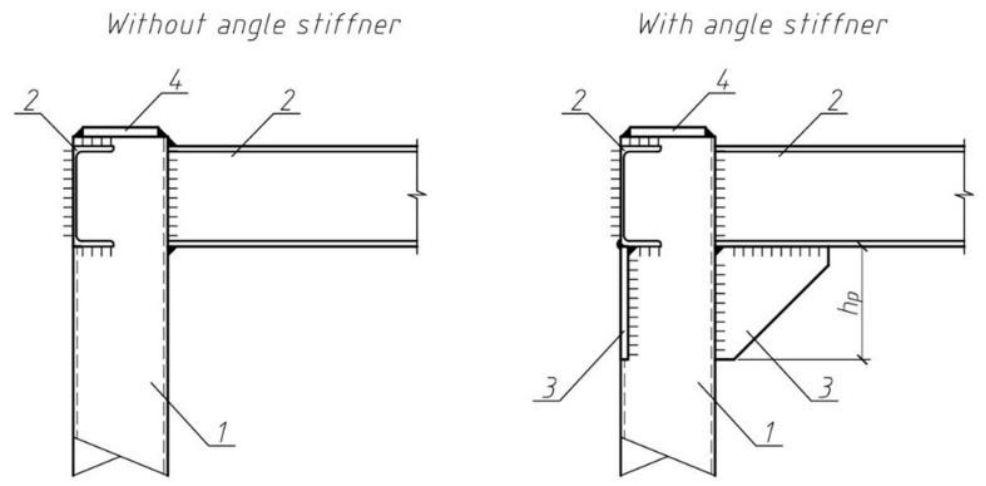

Fig. 2. Node scheme: 1 - rack; 2 - crossbar; 3 - rib; 4 - plug fitting

According to Eurocode 3, the nodes are classified into three groups according to their rotational stiffness $\mathrm{S}_{\mathrm{j}, \text { ini }}$ : rigid, semi-rigid and hinged (Fig. 3). Rotational stiffness is the moment that causes a single rotation of the node. To determine the stiffness of the fastening, it is necessary to establish the relationship between the rotation angle and the moment in the node. In general case, this dependence is nonlinear, therefore it is necessary to determine the limiting moment $\mathrm{M}_{\mathrm{j}, \mathrm{Rd}}$, which can support the element attached to the node, or directly the attachment itself. Initial rotational stiffness $S_{j, \text { ini }}$ is the tangent of the relationship $2 / 3 \cdot \mathrm{M}_{\mathrm{j}, \mathrm{Rd}}$ to the rotation angle of the section at a given value of the moment. The limiting values of the rotational stiffness for rigid and hinged fastenings are determined depending on the bending stiffness of the adjoining elements. The conditions for the classification of fasteners according to Eurocode 3 are as follows for a rigid, semi-rigid and articulated joint, respectively:

$$
\begin{gathered}
S_{j, i n i}>S_{j, R}=k_{b} \cdot E \cdot I_{b} / L_{b} \\
S_{j, R}>S_{j, i n i}>S_{j, P} \\
S_{j, \text { ini }}<S_{j, P}=0,5 \cdot E \cdot I_{b} / L_{b}
\end{gathered}
$$

where $S_{j, R}, S_{j, P}$ are the limiting values of rotational stiffness for rigid and hinged joints, respectively;

$E$ is the elastic modulus of steel;

$I_{b}$ is a moment of inertia of the girder (fastening element);

$L_{b}$ is a deadbolt span;

$k_{b}$ is the coefficient taking into account the presence of vertical links. For the considered modular blocks $k_{b}=25$. 


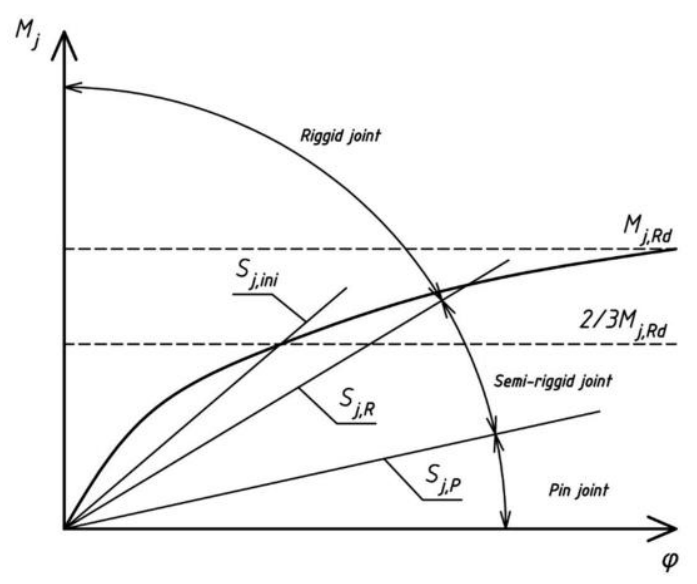

Fig. 3. Node classification graph according to dependency M- $\varphi$

To determine the initial rotational stiffness through a numerical experiment, the finite element method can be used (FEM) [7, 19-22]. It is possible to calculate the necessary parameters to determine $S_{\mathrm{j}, \text { ini }}$ via FEM. However, the construction of the design scheme in FEM is a rather laborious process, while for each node variant it is necessary to create a new model. The specified drawback is not present in the finite element method component (CFEM), implemented in the software package IDEA StatiCA [23, 24].

IDEA StatiCA implements the following types of calculations: determination of the stress-strain state (SSS) of the joint, the calculation of the joint components' stability according to Eurocode, the calculation of the ultimate moment (formation of a plastic hinge), the calculation of the ultimate load on the joint and the element attachment stiffness calculation. In this study, the last calculation mode is of interest. With it, it is possible to determine the rotational stiffness of the bolt attachment to the rack. The software complex calculates $\mathrm{S}_{\mathrm{j}, \text { ini }}$ independently according to the actual deformations and forces determined in the calculation process and in accordance with Eurocode 3.

Design model of the node for IDEA StatiCA is shown in Figure 4.

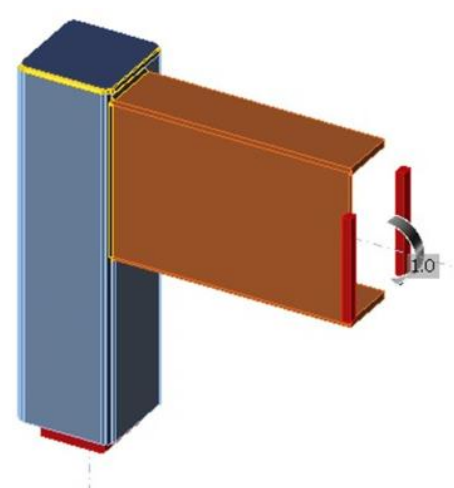

a.

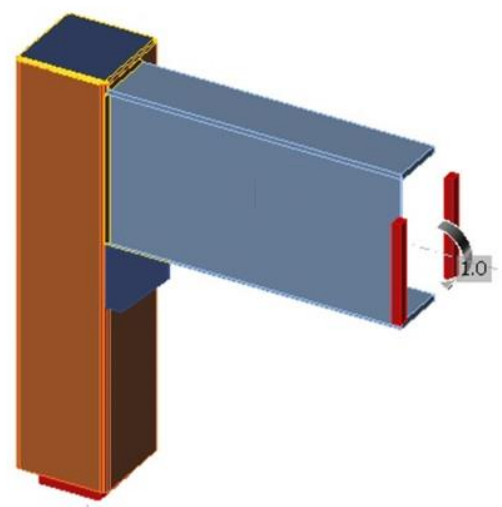

b.

Fig. 4. Node model for IDEA StatiCA: $a$ - without a rib; $b$ - with a rib

In the design model, the rack is a supporting element; it has fixings at the bottom in all six directions. The crossbar is a calculated element, its movements are limited by the vertical plane. The fastening welds are applied on one side along the outer perimeter of the channel. A single bending moment is applied to the girder $\mathrm{M}_{\mathrm{Ed}}$. In this case $\mathrm{M}_{\mathrm{Ed}}$ sets the 
direction of deformation, calculation of the limiting moment $\mathrm{M}_{\mathrm{j}, \mathrm{Rd}}$ is performed by the software package in the course of the calculation.

The studies were carried out for the nodal joints obtained by combining a rack from a square pipe and a girder from a rolled channel. Variants of the connected elements' combinations are presented in Table 1. The studies were carried out for two cases - a seat without an edge and a node with an edge. In the studies, the rib is assumed to be $4 \mathrm{~mm}$ thick. The length and width of the rib were taken to be the same $\left(h_{p}\right)$ and varied from 40 $\mathrm{mm}$ to $160 \mathrm{~mm}$ in $10 \mathrm{~mm}$ increments.

\section{Results}

As a result of the study, the rotational stiffness values of fastening the crossbars to the columns were obtained. The calculated values $\mathrm{S}_{\mathrm{j}, \text { ini }}$ are given in Table. 1 .

Table 1. Rotational stiffness values $\mathrm{S}_{\mathrm{j}, \mathrm{ini}},[\mathrm{MN} \cdot \mathrm{m} / \mathrm{rad}]$

\begin{tabular}{|c|c|c|c|c|c|c|c|c|c|c|}
\hline \multirow{2}{*}{\multicolumn{2}{|c|}{$\begin{array}{c}\text { Framework } \\
\text { element }\end{array}$}} & \multicolumn{9}{|c|}{ Girder } \\
\hline & & {$[12$} & {$[14$} & {$[16$} & {$[18$} & {$[20$} & {$[22$} & {$[24$} & {$[27$} & {$[30$} \\
\hline \multirow{12}{*}{ 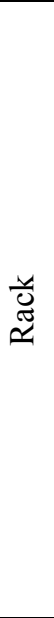 } & $80 \times 4$ & 1.6 & 2.3 & 3.0 & 5.5 & 14.2 & - & - & - & - \\
\hline & $80 \times 5$ & 2.2 & 3.2 & 4.6 & 8.3 & 22.9 & - & - & - & - \\
\hline & $80 \times 6$ & 3.0 & 4.4 & 6.5 & 8.7 & 46.1 & - & - & - & - \\
\hline & $90 \times 4$ & 1.5 & 2.2 & 2.6 & 3.9 & 5.9 & 11.0 & $\infty$ & - & - \\
\hline & $90 \times 5$ & 2.1 & 2.9 & 3.8 & 5.5 & 8.4 & 16.8 & $\infty$ & - & - \\
\hline & $90 \times 6$ & 2.8 & 3.9 & 5.1 & 7.4 & 11.5 & 24.2 & $\infty$ & - & - \\
\hline & $100 \times 4$ & 1.5 & 2.1 & 2.4 & 3.5 & 4.8 & 8.6 & 10.7 & 71.2 & $\infty$ \\
\hline & $100 \times 5$ & 2.1 & 2.9 & 3.5 & 4.8 & 6.5 & 9.1 & 16.7 & 83.1 & $\infty$ \\
\hline & $100 \times 6$ & 2.8 & 3.7 & 4.6 & 6.3 & 8.7 & 11.6 & 23.6 & $\infty$ & $\infty$ \\
\hline & $120 \times 4$ & 0.6 & 2.2 & 2.4 & 3.2 & 4.2 & 5.1 & 6.1 & 9.1 & 13.6 \\
\hline & $120 \times 5$ & 1.0 & 2.9 & 3.4 & 4.4 & 5.6 & 6.9 & 8.6 & 12.9 & 20.2 \\
\hline & $120 \times 6$ & 1.4 & 3.8 & 4.4 & 5.7 & 7.1 & 8.7 & 11.3 & 16.9 & 28.7 \\
\hline
\end{tabular}

To classify the node type, the rotational stiffnesses values, given in Table. 1, should be compared with the bending stiffness of the girders, which depends on their span. From formula (1), the minimum values of the spans at which the nodal connection is considered rigid, can be derived:

$$
L_{b}>k_{b} \cdot E \cdot I_{b} / S_{j, i n i}
$$

It was found that the stiffness degree of the assembly strongly depends on the crossbar span. The larger the span of the girder, the brighter the stiffness of the joint is manifested. Table 2 shows the calculated values of the girder minimum spans at which the node can be considered rigid. In table 2 , for the elements with a conventionally infinite rotational stiffness of the fastening, the minimum span is conventionally equal to zero, such a connection can always be considered rigid. For most of the options considered, a rigid connection is provided when the girder span is more than $6 \mathrm{~m}$. The exception is the nodal connections, in which the width of the channel flange is close to the width of the rack section. In this case, the pipe walls work as stiffeners, this leads to a significant decrease in the girders rotation angles and an increase in the rotational stiffness $S_{j, i n i}$. Modular buildings 
are characterized by the spans in the interval $2.4 \div 6 \mathrm{~m}$, less often up to $9 \mathrm{~m}$, therefore, nodes with direct abutment of channels to columns made of square pipes should be considered in the general case "almost rigid". An almost rigid connection is a bond of ultimate stiffness. The magnitude of this stiffness can be determined numerically, for example, using FEM. When designing modular buildings, it is recommended to take into account the final stiffness of the connections in the design models.

Table 2. Minimum girder spans for rigid nodes, [m]

\begin{tabular}{|c|c|c|c|c|c|c|c|c|c|c|}
\hline \multirow{2}{*}{\multicolumn{2}{|c|}{ Element }} & \multicolumn{9}{|c|}{ Girder } \\
\hline & & {$[12$} & {$[14$} & {$[16$} & {$[18$} & {$[20$} & {$[22$} & {$[24$} & {$[27$} & {$[30$} \\
\hline \multirow{12}{*}{$\begin{array}{l}\text { ỹ } \\
\text { ¿゙ }\end{array}$} & $80 \times 4$ & 10.1 & 11.3 & 13.2 & 10.5 & 5.7 & - & - & - & - \\
\hline & $80 \times 5$ & 7.3 & 8.1 & 8.6 & 6.9 & 3.6 & - & - & - & - \\
\hline & $80 \times 6$ & 5.4 & 5.9 & 6.1 & 6.6 & 1.8 & - & - & - & - \\
\hline & $90 \times 4$ & 10.7 & 11.8 & 15.2 & 14.7 & 13.7 & 10.2 & 0 & - & - \\
\hline & $90 \times 5$ & 7.7 & 9.0 & 10.4 & 10.5 & 9.6 & 6.7 & 0 & - & - \\
\hline & $90 \times 6$ & 5.8 & 6.7 & 7.8 & 7.8 & 7.0 & 4.6 & 0 & - & - \\
\hline & $100 \times 4$ & 10.7 & 12.4 & 16.5 & 16.4 & 16.8 & 13.0 & 14.3 & 3.1 & 0 \\
\hline & $100 \times 5$ & 7.7 & 9.0 & 11.3 & 12.0 & 12.4 & 12.3 & 9.2 & 2.7 & 0 \\
\hline & $100 \times 6$ & 5.8 & 7.0 & 8.6 & 9.1 & 9.3 & 9.6 & 6.5 & 0 & 0 \\
\hline & $120 \times 4$ & 26.7 & 11.8 & 16.5 & 17.9 & 19.2 & 21.9 & 25.1 & 24.2 & 22.6 \\
\hline & $120 \times 5$ & 16.1 & 9.0 & 11.6 & 13.1 & 14.4 & 16.2 & 17.8 & 17.1 & 15.2 \\
\hline & $120 \times 6$ & 11.5 & 6.9 & 9.0 & 10.1 & 11.4 & 12.8 & 13.6 & 13.0 & 10.7 \\
\hline
\end{tabular}

Fig. 5 shows a graph of the limiting rotational stiffness of fastening the girder from the channel 20 to the columns of various sections. The solid line shows the minimum rotational stiffness $S_{j, R}$, at which the node can be considered rigid. The horizontal lines show the rotational stiffnesses calculated in IDEA StatiCA. According to the condition (1), a node is rigid if the horizontal line lies above the curve $S_{j, R}$. It can be seen from the graph that only the connections of the girder with the uprights made of pipes $80 \times 6,80 \times 5$ and $80 \times 4$ can be considered rigid with a span of less than $6 \mathrm{~m}$. With an increase in the dimensions of the rack, the rotational stiffness is greatly reduced. The ratio of rotational stiffness shown in the graph is typical for all the considered connection options.

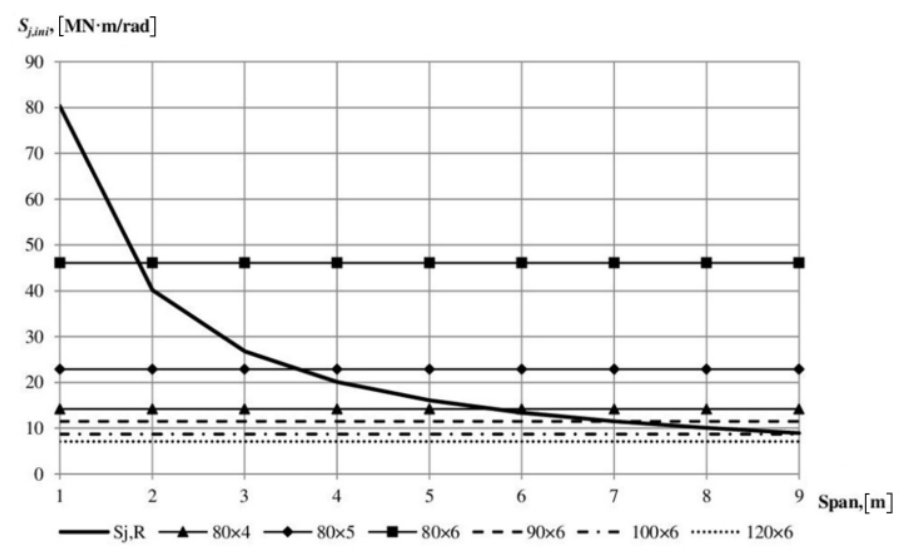

Fig. 5. Boundary rotational stiffness for channel girder 20 
The following is a study of the rib sizes' effect on the joint rotational stiffness value. It is known that the minimum joint stiffness decreases following a decrease in the wall thickness of the strut. Therefore, further research was carried out only for the nodes with a strut thickness of $4 \mathrm{~mm}$. Fig. 6 shows the results of a rotational stiffness study of fastening channel 16 with ribs of different sizes.

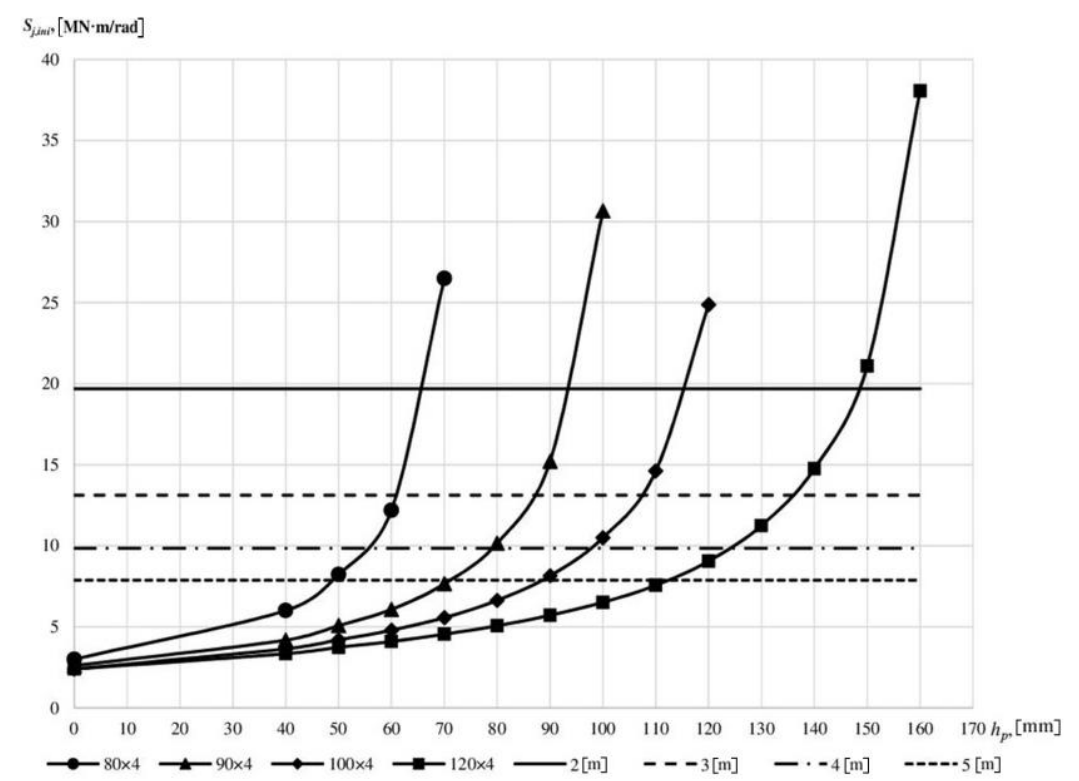

Fig. 6. Rotational stiffness of fastening channel 16 with an edge to racks of various cross-sections

Fig. 6 shows the rotational stiffness dependence graphs on the rib dimensions. The horizontal lines show the minimum values $S_{j, \text { ini }}$ for various spans of the girder, in which the knot is considered rigid. It can be seen in Fig. 6 that the change in the rotational stiffness of the attachment depending on the size of the rib has a non-linear character. When the rib size is close to the size of the rack section, there is a sharp increase in $S_{j, \text { ini }}$. A similar nature of the change is observed for all the considered variants of cross-sections of girders. The only exceptions are the channel 20 joints with a rack $80 \times 4$ and the channel 22 with $90 \times 4$, for which, even with a minimum rib size of $40 \mathrm{~mm}$, values of rotational stiffness are achieved above the minimum corresponding to rigid nodes.

The stiffeners size to ensure a rigid abutment of the channel to the square pipe depends both on the cross-section of the rack and on the cross-section of the girder. At the same time, it is difficult to establish a direct relationship between these parameters. Therefore, a nomogram has been drawn up with the minimum dimensions of the ribs for the considered cross-sections of the girders, depending on the cross-sections of the racks to which they are attached (Fig. 7). At the ribs sizes the node shown in Fig. 7 can be considered rigid. 


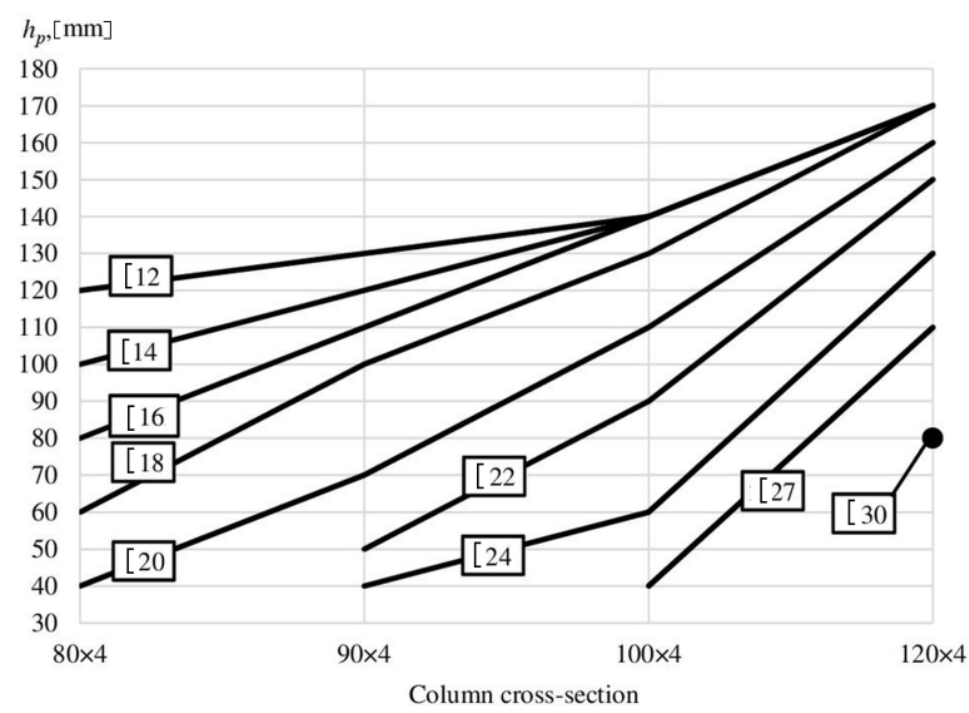

Fig. 7. Rib sizes for rigid nodes

\section{Conclusion}

According to the study, the following conclusions can be formulated.

1. Welded UPN channel joints with pipe studs without stiffeners are semi-rigid with spans typical of modular buildings. The exception is the joints in which the width of the cross-section is close to the width of the girder cross-section, in which case the column walls are included in the work as stiffeners, which leads to a significant decrease in the cross-section rotation angle of the girder cross-section. In the considered variants of the nodes, this is observed in the joints of channels 24 with uprights from the pipes $90 \times 4,90 \times$ $5,90 \times 6$, channels 27 with a pipe $120 \times 6$, as well as 30 with the pipes $100 \times 4,100 \times 5$, $100 \times 6$. These connections can be considered absolutely rigid.

2. It is recommended to arrange stiffening ribs to ensure a rigid junction of the channel girder to the square pipe rack. The minimum dimensions of the stiffeners should be taken according to Fig. 7.

Within the framework of this study, the parameters of the modular buildings' nodal connections, in which the nodes behave as rigid, have been determined.

\section{References}

1. A. Dorrhofer, M. Rsenthal, G. Staib, Components and Systems: Modular Constructions. Design, Structure, new Technologies, Basel-Boston-Berlin: Institut für internationale Architektur-Documentacion Gmbh \& Co. KG, 2008.

2. G.M. Badyin, S.A. Sychev, Modern problems of science and education 2-1 (2015).

3. O.N. Beskorovainaya, D.S. Bychkov, Z.A. Gaevskaya, Construction of unique buildings and structures 1(16), 61-71 (2014).

4. A.N. Mushinsky, S.S. Zimin, Construction of unique buildings and structures, 4(31) 182-193 (2015). 
5. V.S. Shirokov, A.V. Soloviev, Urban planning and architecture 8 (1), 24-27 (2018).

6. V.M. Tusnina, V.D. Platonova, Industrial and civil engineering 9, 28-33 (2020).

7. V.Yu. Alpatov, A.O. Lukin, A.A. Sakharov, Industrial and civil engineering 9, 9-14 (2015).

8. V.S. Shirokov, V.Yu. Alpatov, E.A. Gordeev, Vestnik MGSU 16 (1), 20-29 (2021).

9. I.I. Vedyakov, O.V. Korzhov, B.S. Tsetlin, O. G. Prilutsky, Industrial and Civil Engineering 6, 20-23 (2009).

10. I.S. Kholopov, V.S. Shirokov, A.V. Soloviev, Bulletin of the Lipetsk State Technical University 4, 56-62 (2015).

11. Bong-Ho Cho, Jae-Sub Lee, Hongjin Kim, Dae-Jin Kim, Applied Sciences 9, 1929 (2019).

12. Andrew William Lacey, Wensu Chen, Hong Hao, Kaiming Bi, Engineering Structures. 198, 109465 (2019).

13. Andrew William Lacey, Wensu Chen, Hong Hao, Kaiming Bi, Engineering Structures. 227, 111409 (2020).

14. J.Y.R. Liew, Z. Dai, Y.S. Chua, Steel concrete composite systems for modular constructions of high-rise buildings, 12th International Conference on Advances in Steel-Concrete Composite Structures (ASCCS 2018), Universitat Politècnica de València, València, Spain, 2018.

15. Sze Dai PANG, J Y Richard Liew, Ziquan Dai, Yanbo Wang, Prefabricated prefinished volumetric construction joining techniques review, 2016 Modular and Offsite Construction (MOC) Summit.: Edmonton, Alberta, Canada, 2016.

16. En-Feng Deng, Liang Zong, Yang Ding, Zhe Zhang, Jun-Feng Zhang, Feng-Wei Shi, Li-Ming Cai, Shu-Cai Gao, Thin-Walled Structures 155, 106924 (2020).

17. Andrew William Lacey, Wensu Chen, Hong Hao, Kaiming Bi, Engineering Structures 213, 110628 (2020).

18. Andrew William Lacey, Wensu Chen, Hong Hao, Kaiming Bi, Journal of Building Engineering 16 45-56 (2018).

19. V.V. Nadolskiy, Bulletin of Polotsk State University. Series F. Construction. Applied Science 8, 121-130 (2018).

20. V.V. Ryumin, Modern building structures made of metal and wood 16, 216-223 (2012).

21. I.S. Kholopov, V.Yu. Alpatov, A.V. Atamanchuk, Building materials, equipment, technologies of the XXI century 1, 66-68 (2008).

22. S.A. Sokolov, A.N. Kachaun, P.O. Skudalov, S.V. Cheremnykh, Bulletin of the Tver State Technical University. Series: Construction. Electrical and chemical engineering 2 (2), 36-42 (2019).

23. L. Sabatka, F. Wald, J. Kabelac, D. Kolaja, M. Pospisil, J. of Civil Engineering and Architecture 9, 895-901 (2015).

24. R.V. Baroev Analysis of steel structures joints by the component finite element method, Information on http://isicad.ru/ru/articles.php?article_num=20749. 